\title{
COMPETENCIAS FUNDANTES: SENTIDO DE AUTOAPROPIACIÓN Y SENTIDO COMUNITARIO
}

\author{
Lourdes Lavaniegos González*
}

\section{Resumen}

El diseño curricular por competencias parte de la consigna de preparar a las personas para que sean capaces de enfrentar los problemas que la vida les presentará, y se reduce casi siempre a dos círculos de acción: el académico y el laboral.

Todo proyecto educativo supone el desarrollo de la individualización y la socialización como fines esenciales y el enfoque por competencias no sólo los considera como parte de su perfil de egreso; sino como base para la actividad educativa.

Entonces son requisitos para las competencias académicas dos competencias "fundantes": el sentido de autonomía y el sentido comunitario que no se reducen a la simple autoestima y a tener un nivel de comunicación armónica con los demás; sino que llegan a la profundidad del ser humano formando un círculo virtuoso.

El cometido de esta investigación es describir la importancia de las competencias fundantes, sus contenidos educativos, los obstáculos que enfrentan en la actualidad y la entretejida relación que existe entre ambos. Por último se confrontan los hallazgos teóricos recabados con el perfil de egreso del currículum por competencias del plan de estudios de secundaria por considerarse el más actualizado en nuestro país.

En sus siguientes fases, este trabajo se propone identificar sujetos con un alto desarrollo de competencias fundantes para descubrir elementos comunes en su vida familiar que puedan servir de base para una propuesta educativa para las familias mexicanas.

*Licenciatura en Pedagogía por la Universidad Panamericana, Maestría en Educación por la Universidad Abierta de S.L.P., Estudiante del Doctorado en Ciencias para la Familia de Enlaces Educativos, Estudios en el área de Educación Perinatal y Formación de docentes lasallistas, En el ámbito laboral ha fungido como Coordinadora de Primaria en el Colegio Miraflores, D.F., Directora de Primaria en Liceo del Nuevo Mundo, Pachuca, Hgo., Directora de la Escuela de Ciencias Humanas de la Universidad La Salle Pachuca., Actualmente soy 
Directora Técnica del Colegio Montessori (Secundaria) y docente en la Universidad La Salle Pachuca en la Coordinación de Humanidades, la Escuela de Ciencias Humanas y la Dirección de Educación Continua. lourdeslavaniegos@yahoo.com.mx

\section{Abstract}

The curricular design by competitions parts from the slogan to prepare people so that they are able to face the problems that life will present to them, and it is usually reduced to two circles of action: academic and the working one.

Every educative project supposes the development of individualisation and socialization as essential aims and the approach by competences considers them not only as part of its graduation profile; but as the base for the educative activity.

Then two "foundation" academic competences are requisite for the academic competences: the sense of autonomy and the communitarian sense that are not reduced to simple self-esteem and having a level of harmonic communication with others; but they go to the depth of the human being forming a virtuous circle.

The assignment of this investigation is to describe the importance of the foundation competences, their contents, the obstacles they face at present and the interlaced relation that exists between both. Finally, the successfully obtained theoretical findings with the graduation profile of the curriculum by competences are confronted with the curriculum of secondary school, considered the most updated in our country.

In its following phases, this paper proposes to identify subjects with a high development of "foundation" competences to discover common elements in its familiar life so they can serve as base for an educative proposal for Mexican families.

PALABRAS CLAVE: Competencias, sentido de autoapropiación, sentido comunitario, plan de estudios secundaria 2006

\section{Introducción}

Los cometidos más importantes de la educación, independientemente de la corriente filosófica de la cual han surgido, pueden resumirse en dos: la individualización y la socialización que se relacionan en una dinámica que requiere de ambos elementos para su equilibrio.

En los últimos tiempos, la búsqueda de la individualización se ha enfatizado, y en cambio, la socialización se ha reducido a simples habilidades de comunicación y convivencia que evitan el compromiso comunitario. 
La familia es el ámbito en que la educación de un ser humano comienza y tiene un peso mucho mayor que aquel que llegarán a tener los muchos años de escuela formal; pero en la familia actual, se observa la misma tendencia: transigir con las diferencias individuales y en contrapartida, disminuir la exigencia de habilidades de socialización, como pueden ser la participación en las labores del hogar o la costumbre de ser generoso con los propios bienes.

Por otro lado en el enfoque de desarrollo curricular por competencias que se utiliza actualmente para el diseño de planes de estudio, se enfatizan tanto la autonomía como el sentido comunitario. Si la familia ha hecho su trabajo, el estudiante llegará a la escuela con los requisitos mínimos para obtener resultados satisfactorios bajo dicho enfoque.

La presente investigación se limita por el momento a describir qué implicaciones tienen ambos cometidos considerados como competencias vitales o fundantes: la competencia para el sentido de autonomía y la competencia para el sentido comunitario, porque se ubican necesariamente sobre las competencias académicas vertidas en los actuales planes de estudio.

Fines de la educación

La educación consiste en: "....aprender a descubrir, a reconocer y a ope-rativar las innatas potencialidades de la propia actividad consciente; así como las condiciones que hacen más probable irlas liberando de los impedimentos que obstaculizan su desarrollo positivo... Tal proceso bien puede denominarse "Autoapropiación"...exige e implica el conocimiento de sí mismo y del entorno sociocultural" (Avilez 2006, p.64-65).

De donde se deducen tres condiciones: la educación como un proceso inacabado, y la doble misión: de crecimiento personal y de relación con lo que se encuentra más allá de lo personal.

La competencia por su lado, puede definirse como: "...la capacidad que tiene un individuo para realizar una tarea dada, o como un nivel de ejecución o dominio que los ciudadanos requieren para desempeñarse adecuadamente en la sociedad en la que viven" (Medina 1996, p.81).

Nuevamente resaltan tres condiciones: un nivel de dominio mínimo, la capacidad inherente al individuo y su adecuado desempeño social.

Se observa en consecuencia un triple paralelismo que permite afirmar que el sentido de autonomía y el sentido comunitario son las competencias que persigue como fin último todo proceso de desarrollo del ser humano. 
La educación es un proceso continuo e inacabado cuyo nivel de dominio crece de manera continua. La educación tiene una vertiente individual, de crecimiento personal que puede llamarse "autonomía" o según el término de Avilez: "autoapropiación" y que otorga al sujeto capacidad de acción. La educación tiene también una vertiente social que le lleva a relacionarse con lo que está fuera de sí mismo y con los que están fuera de sí mismo.

La doble finalidad no puede esquivarse; se encuentra entrelazada en: "La exigencia de la libertad del individuo, por un lado, y la exigencia de imposición de obligaciones desde las estructuras sociales por otro." (Durr 1971,21)

Sentido de autopropiación

El sentido de autoapropiación puede explicarse como la competencia que engrana los conocimientos, habilidades, valores y actitudes necesarios para la inividualización y que se encamina como fin último a la conciencia de dignidad.

En los últimos tiempos, a diferencia de épocas anteriores, se ha reconocido la necesidad de desarrollar este sentido en los sujetos, existe conciencia de los derechos humanos y hay una declaración de los derechos infantiles firmada por un gran número de países entre los que se encuentra el nuestro. En el campo psicológico se habla de diferencias caractereológicas, multiplicidad de inteligencias y variedad de tipos mentales y en consecuencia se consideran las divergencias personales no sólo como posibles, sino como enriquecedoras y deseables. En el campo sociológico, el advenimiento de los ideales democráticos ha favorecido que uno de los principales valores de la época sea la aceptación de la diversidad y la tolerancia a los diferentes puntos de vista.

Pero el sentido de autoapropiación puede tomar matices mucho más profundos que la simple sensación de autoestima. La autoapropiación, según la percepción de Lonergan (en Pérez Valera s/f, p. 21) "Se trata de uno mismo, tomando posesión de sí mismo" y para ello se requiere pasar por cinco niveles:

- Nivel de la experiencia: supone ponerse en contacto con la vivencia de nuestra interioridad, saber qué es lo que pasa en nuestro interior.

- Nivel de intelección: Se trata de recordar y de entender, es cuando llega un chispazo intelectual (insight) que nos permite hacer conciencia de que hemos comprendido algo.

-Nivel de juicio: "Es caer en la cuenta de que hay actos de entender que están equivocados" (Pérez s/f, p. 13). Conforme más vamos comprendiendo, existen mayores probabilidades de darnos cuenta que nuestros juicios anteriores eran equivocados o al menos incompletos. 
-Nivel de decisión: Supone deliberación y conlleva la inclusión de valores que permiten una toma de posición ante lo que se ha experimentado, comprendido y juzgado.

- Nivel del amor sin fronteras: se integra a partir de los niveles anteriores y supone la sublimación de valores a través del amor: "...sublima todas las demás operaciones de la conciencia individual humana". (Pérez s/f, p. 30) Para Lonergan, el ser humano que no llega a

este nivel queda truncado porque no puede reunir en sí una plena intencionalidad y por tanto no puede llegar a ser verdaderamente "auténtico", no se autoposee.

Para poder dar al sentido de autoapropiación condición de "competencia" se requiere enumerar los conocimientos, habilidades, valores y actitudes que la integran. A partir de los cinco niveles de Lonergan puede concluirse que:

1. Los conocimientos mínimos que integran la competencia de sentido de autoapropiación pueden reducirse a la comprensión teórica de los cinco niveles.

2. Las destrezas consisten en reconocer los procesos que sigue la mente al paso por dichos niveles. Si bien hay rasgos generales, las diferencias individuales han de ser constatadas.

3. Los valores serían de índole personal: autoestima, autodominio, curiosidad.

4. Las actitudes necesarias consistirían en la reflexión y la adaptación al cambio.

Sentido comunitario

El sentido comunitario se considera como la competencia que engrana los conocimientos, habilidades, valores y actitudes necesarios para la socialización y en último término encarna la capacidad de "servir". Savater afirma que al contrario de los animales, el ser humano no tiene un cuerpo adaptado a ninguna especialización, esto lo hace vulnerable, pero al mismo tiempo le permite salir de la determinación que viven el resto de los seres vivos. Sólo existe para nosotros una determinación: "No estamos determinados a vivir en ningún paisaje ni en ningún clima, pero sí a convivir con semejantes que nos enseñen y ayuden. El medio ambiente natural específico de los seres humanos es la sociedad." (Savater 2003, p. 26-27). 
Enseñar y aprender, ayudar y ser ayudados; este es el fundamento del sentido comunitario. Que en nuestra época se ha visto obstaculizado en mayor medida que en las precedentes por megatendencias (CELAM 2000, p. 34-66) de origen socioeconómico fácilmente constatables en los países latinoamericanos:

- Medios de comunicación de amplio impacto que favorecen la transmisión de formas culturales consumistas y egoístas.

- Distribución inequitativa de los ingresos que acarrea contrastes entre las grandes fortunas y los cúmulos de población en extrema pobreza incapaces de un entendimiento común.

- Ideales democráticos dependientes de criterios financieros que provocan la corrupción y la falta de credibilidad.

- Procesos de secularización que admiten la diversidad de creencias y

valores y en consecuencia la desintegración social.

- Educación de baja calidad que no permite el desarrollo de competencias necesarias para la adaptación exitosa a la sociedad.

- Desintegración familiar en que las formas de convivencia se minimizan impactando en el ejercicio de la primera experiencia comunitaria.

- Deterioro ambiental que pone en riesgo la viabilidad de la vida y coadyuva al empobrecimiento de las poblaciones marginadas.

- Replanteamiento del papel de la mujer que influye en la calidad de la

vida familiar dado que no se ha previsto un rol sustitutivo.

En circunstancias tan adversas, resulta indispensable sugerir un itinerario para que la educación del sentido comunitario se encamine en última instancia al "servicio":

- Cobertura de las propias necesidades: porque no puede darse lo que no se tiene, en primer lugar el ser humano requiere reconocer sus necesidades básicas cubiertas.

- Intuición de las necesidades de los demás: una vez cubierto lo mínimo indispensable, el ser es capaz de voltear hacia los demás. 
- Participación en las labores de mantenimiento material.

- Colaboración con la salud del clima grupal.

Cabe aquí tomar conciencia de que el primer paso se encuentra, al menos en teoría, cubierto a partir de la orientación global hacia la defensa de los derechos humanos; sin embargo, la responsabilidad hacia los demás, se diluye debido a las megatendencias mencionadas.

Imaginemos un panorama en que un niño pequeño hace un berrinche porque quiere una galleta, se cumple su capricho y al final queda la mitad hecha migajas en el suelo. Ahora tratemos de evocar otro ambiente en que un niño de la misma edad quiere la galleta, se le orienta para que la pida adecuadamente, tal vez hasta se le pida que la comparta y al final se le enseña a recoger los restos. Como puede observarse, la formación de la competencia de sentido comunitario no depende tanto de la edad, como de la intención educativa.

Para poder considerar el sentido comunitario como competencia, puede resumirse que busca complementar lo siguientes elementos:

- En cuanto a conocimientos: conocerse uno entre muchos otros.

- En cuanto a destrezas: la intuición sobre los que ocurre con los

demás, y la habilidad para postergar el goce personal.

- En cuanto a valores: generosidad, laboriosidad, tolerancia.

- En cuanto a actitudes: observación y adaptación a la realidad.

Relación entre sentido de autoapropiación y sentido comunitario

¿Qué va primero la formación del sentido de autoapropiación o la del sentido comunitario? ¿tienen ambas el mismo peso? Resulta evidente reconocer que se complementan porque el ser humano es "ser social" $y$

en ese sólo concepto se observa ya la realidad individual y colectiva de su esencia. Sin embargo, la concepción ética como afirma Adela Cortina, ha ido caminando de lo individual a lo colectivo, de lo intrasubjetivo a lo intersubjetivo por tres razones: en primera instancia los resultados de nuestras acciones no son siempre las previstas y nuestras decisiones personales se unen a las de otros, en segundo lugar nuestra libertad no tendría ningún sentido si no pudiera expresarse en un contexto social y en último término los descubrimientos racionales individuales sólo están completos cuando se comparan con los de los demás. (Cortina 1996, p.53) 
Sin embargo, la educación, no puede quedarse en una simple sensación de autoestima, o en la ilusión de que las relaciones superficiales son suficientes. Para el logro de las competencias del sentido de autoapropiación y del sentido comunitario deben salvaguardarse seis condiciones (Ortolani 2000, p.96-97):

- Individuación personal con todos los valores de subjetividad, en

contra de la actual tendencia a la masificación.

- Contacto social con otros seres humanos en contra del peligro de aislamiento (provocado por los avances tecnológicos).

- Sana independencia para que cada quien realice su propio esfuerzo personal guardando cierta "distancia" de los demás en contra de la tendencia a la dependencia excesiva.

- Experiencias profundas con personas significativas para llegar a la integración personal, en lugar del desprecio de los demás.

- Igualdad y democracia en contra de la tendencia a mantener privilegios e injusticias.

- Dignidad del hombre en contra de la tendencia al escepticismo y a la falta de esperanza.

La primera condición es seguramente el desarrollo de la autoapropiación, pero casi de inmediato debiera aparecer el sentido comunitario para propiciar una dinámica continua, mientras más me apropio de mí misma, más puedo colaborar con mi comunidad; pero mientras más colaboro con mi comunidad, más me conozco y me apropio de mí misma

Para fines de estudio resulta difícil determinar el límite entre la sana autoestima y el orgullo egoísta; en consecuencia, parece que lo más viable es identificar los elementos de la competencia para el sentido comunitario que necesariamente incorporan los elementos de autoapropiación según la explicación dinámica que se ha hecho en las líneas anteriores.

Relación entre competencias fundantes

Y competencias académicas 
El enfoque de desarrollo curricular por competencias, debiera provocar una transformación radical en la educación si se comprendieran cabalmente los supuestos que acarrea. Dicho enfoque tiene la ventaja de encarar el conocimiento como un todo integrado en que se aglutinan informaciones, habilidades y valores, observando la totalidad del ser humano y preocupándose más por los resultados aplicables a la vida que por el saber de corte enciclopédico.

Parece sencillo, pero trataremos de visualizar cómo basa su éxito en el sentido de autoapropiación y en el sentido comunitario.

El caso del Plan de Estudios para la Escuela Secundaria 2006, vigente actualmente en México, es el más moderno esfuerzo por incorporar un currículo por competencias en nuestro país; por tanto lo tomaremos como base para el análisis de las competencias fundantes de referencia.

El plan de estudios referido propone cinco tipos de competencias en su perfil de egreso (SEP 2006, p.11-12):

- Competencias para el aprendizaje permanente: suponen asumir la responsabilidad del propio aprendizaje. Requieren los cinco niveles de autoapropiación para a partir de una adecuada metacognición seguir aprendiendo de manera continua; también necesitan socializar lo aprendido para completar el ciclo de racionalización.

- Competencias para el manejo de información. Se refieren al manejo lógico de diversas disciplinas. Se desprenden nuevamente de una adecuada autoapropiación y de la comprensión de la realidad contextual.

- Competencias para el manejo de situaciones. Implican el diseño de un proyecto de vida para el cual es indispensable el conocimiento personal y la posibilidad de interacción con los otros - Competencias para la convivencia. Buscan la relación armónica con los demás que sería imposible si no existe una relación armónica intrasubjetiva. 
- Competencias para la vida en sociedad. Se encaminan a desarrollar el juicio crítico ante los valores y normas sociales para lo cual se re quiere la toma de una posición personal y la adecuación a la realidad social específica.

\section{Conclusiones}

Bajo el modelo de educación formal basado en competencias es muy probable que si la familia ha hecho su trabajo, existan mayores probabilidades de que los resultados sean exitosos dado que en la convivencia familiar adecuada se reúnen dos mecanismos: en un primer nivel, el control homeostático en una estructura temporoespacial con roles específicos y en un segundo nivel, una red de comunicaciones (Estrada 2006, 12). Mecanismos que permitirán al niño llegar a la sociedad habiendo practicado la individualidad en un rol específico y la socialización mediante una comunicación apropiada.

Las competencias fundantes que se han analizado tienen como finalidad última que el educando en formación adquiera conciencia de dignidad y practique el servicio a los demás.

La investigación que se propone, se basa en el supuesto de que un joven que ha aprendido a "servir", será mucho más capaz de convivir adecuadamente y al sentirse útil, elevando consecuentemente su conciencia de dignidad. Como resultado de estos dos logros, estará mejor encaminado a obtener resultados favorables al enfrentarse a un currículum por competencias.

Al considerarse que existe una dinámica de equilibrio entre ambos elementos y que la conciencia de dignidad es más difícil de detectar que la práctica del servicio; se espera poder seleccionar a sujetos que son reconocidos por la comunidad escolar como aquellos capaces de servir a los demás e identificar los elementos que tienen en común en su educación familiar para desprender de ahí una propuesta educativa que pueda ser de utilidad a las familias mexicanas.

\section{Fuentes de consulta}

AVILEZ Espejel, Ricardo José, (2006) La búsqueda humanizante. Lupus Magíster. México.

CELAM, (2000) El tercer Milenio como Desafío Pastoral: Informe CELAM 2000. CELAM. Bogotá. 
CORTINA, Adela, (1996) El quehacer ético: Guía para la educación moral. Santillana. Madrid.

DÜRR, Otto, (1971) Educación en la Libertad. Rialp. Madrid.

ESTRADA Inda, Lauro, (2006) El ciclo vital de la familia. Debolsillo. México.

MEDINA Martínez, Sara Rosa, (1996) Educación y Modernidad: El Bachillerato en México ante los Desafíos del Tercer Milenio. Universidad Autónoma de San Luis Potosí.

ORTOLANI, Valerio, (2000) Personalidad Ecológica.UIA. México.

PÉREZ Valera, José Eduardo, (s/a) El método cognoscitivo en Bernard Lonergan. UIA. México.

SAVATER, Fernando, (2003) El valor de elegir. Ariel. Barcelona.

SEP, (2006) Plan de Estudios 2006: Educación Básica. Secundaria. Comisión Nacional de Libros de Texto Gratuito. México. 